\title{
Ultrashort Pulsed Laser Ablation of Magnesium Diboride: Plasma Characterization and Thin Films Deposition
}

\author{
Angela De Bonis, ${ }^{1}$ Agostino Galasso, ${ }^{1}$ Antonio Santagata, ${ }^{2}$ and Roberto Teghil ${ }^{1}$ \\ ${ }^{1}$ Dipartimento di Scienze, Università della Basilicata, Via dell'Ateneo Lucano 10, 85100 Potenza, Italy \\ ${ }^{2}$ Istituto di Struttura della Materia, CNR UOS Tito Scalo, C/da S. Loja, 85050 Tito Scalo, Italy \\ Correspondence should be addressed to Roberto Teghil; roberto.teghil@unibas.it
}

Received 20 August 2015; Accepted 22 November 2015

Academic Editor: Bo Tan

Copyright (c) 2015 Angela De Bonis et al. This is an open access article distributed under the Creative Commons Attribution License, which permits unrestricted use, distribution, and reproduction in any medium, provided the original work is properly cited.

\begin{abstract}
$\mathrm{A} \mathrm{MgB}_{2}$ target has been ablated by Nd:glass laser with a pulse duration of $250 \mathrm{fs}$. The plasma produced by the laser-target interaction, showing two temporal separated emissions, has been characterized by time and space resolved optical emission spectroscopy and ICCD fast imaging. The films, deposited on silicon substrates and formed by the coalescence of particles with nanometric size, have been analyzed by scanning electron microscopy, atomic force microscopy, X-ray photoelectron spectroscopy, micro-Raman spectroscopy, and X-ray diffraction. The first steps of the films growth have been studied by Transmission Electron Microscopy. The films deposition has been studied by varying the substrate temperature from 25 to $500^{\circ} \mathrm{C}$ and the best results have been obtained at room temperature.
\end{abstract}

\section{Introduction}

Metal borides films, which show peculiar characteristics at nanoscale level, are currently used in a large number of applications, in particular as corrosion resistant coatings and thermal and diffusion barriers [1]. Among metal diborides, magnesium diboride has attracted great interest due to its superconductivity at about $40 \mathrm{~K}$ [2]. In fact, since $\mathrm{MgB}_{2}$ is the nonoxide material with the highest transition temperature, the deposits of this material in the form of thin films can be very important for many electronic applications. In particular, magnesium diboride thin films have been investigated for applications in high-field magnets, radio frequency cavities used in accelerators, Josephson junctions, and digital circuits [3-5] even if all the most probable applications remain at the moment niche applications and only in the future could this material have good chances to be used in a wider market [6] and many different techniques have been used to produce thin films of this material [3]. In general, one of the most useful techniques to deposit thin films of materials with technological interest, such as metal borides, is Pulsed Laser Deposition (PLD) [7, 8]. PLD has been already used to deposit magnesium diboride thin films but, even if some works concern films produced directly by laser ablation [9-11], in many cases PLD was only the first step of a procedure including ex situ or in situ annealing of the produced deposits, often in Mg rich atmosphere [12-22]. The necessity of a postannealing is not surprising since all the cited ablation-deposition works were performed by using lasers with pulse durations of the order of nanoseconds. In ns PLD the film formation depends strictly on the condensation of atomic species, in this case $\mathrm{Mg}$ and $\mathrm{B}$, on the substrate, followed by their surface diffusion and nucleation. The sticking coefficient of magnesium is in general very low, especially if the substrate temperature is higher than $200^{\circ} \mathrm{C}$, implying that there is only a small probability that it could react with boron to form $\mathrm{MgB}_{2}$ on the surface [11]. The best way to enhance this probability is to increase the flux of $\mathrm{Mg}$ on the surface.

When the PLD is carried out by a laser with pulse duration lower than 1 ps (ultrashort pulse) the mechanism of films formation is very different. In fact, depending on the ablation mechanism, the main constituents of the laser produced plasma, often called plume, are a large number of 
particles with nanometric size and these nanoparticles (NPs) give the larger contribution to the formation of the deposited films $[8,23,24]$. The origin of NPs is still a matter of debate but there is a growing consensus about their origin directly from target fragmentation and ejection of molten material $[8,25-$ 29] even if other hypotheses, such as gas phase reaction, have been also proposed [30]. So, if we consider that in ultrashort PLD condensation from gas phase atomic species is probably only a secondary process in the film formation, the application of this technique to the deposition of magnesium diboride thin films could be useful to minimize the problems related to the low sticking coefficient of $\mathrm{Mg}$.

In this work, with the aim of both verifying ablationdeposition mechanisms already proposed [28, 31, 32] and studying the effect of the substrate temperature on the thin films composition, a target of commercial $\mathrm{MgB}_{2}$ has been ablated in vacuum by $\mathrm{Nd}$ :glass laser with a pulse duration of $250 \mathrm{fs}$. Considering that no data were present in literature about either the plasma produced by fs ablation of magnesium diboride or the films deposited in that way, we have used the techniques already utilized for other systems [28, 31] to characterize the ablation and deposition of $\mathrm{MgB}_{2}$. The plasma produced by the laser-target interaction has been characterized by time and space resolved optical emission spectroscopy and ICCD fast imaging. The films, deposited on silicon substrates, have been analyzed by scanning electron microscopy, atomic force microscopy, X-ray photoelectron spectroscopy, micro-Raman spectroscopy, and X-ray diffraction. The size distribution of the particles forming the films has been studied by Transmission Electron Microscopy. The influence of the deposition parameters on films characteristics has been studied by varying the substrate temperature from 25 to $600^{\circ} \mathrm{C}$. The results have been explained considering an ablation-deposition mechanism involving melted NPs directly ejected from the target.

\section{Experimental}

Ablation and deposition processes were performed by means of an experimental apparatus, already described [32], consisting of a stainless steel vacuum chamber evacuated by a scrollturbomolecular pumping system to a pressure of $1.5 \times 10^{-4} \mathrm{~Pa}$. The chamber was equipped with a support for the target, rotating with a speed of $1 \mathrm{rpm}$ to minimize the craterization effects, quartz windows for the laser beam inlet and the plasma emission detection, and a resistively heated substrate holder. Cold pressed pellets from commercial $\mathrm{MgB}_{2}$ powder (Aldrich) were used as ablation targets. The substrates ((100) oriented silicon wafers from Aldrich) were kept in front of the target at a distance of $2 \mathrm{~cm}$. The deposition substrates were Formvar carbon film coated copper grids only in the case of samples for transmission microscopy analyses. The ablation laser source was a frequency doubled $\mathrm{Nd}$ :glass laser with the following characteristics: $\lambda=527 \mathrm{~nm}, E=3.0 \mathrm{~mJ}$, repetition rate $=10 \mathrm{~Hz}$, and pulse duration $=250 \mathrm{fs}$. The laser beam, focused by a suitable optical system, impinged on the target surface with an angle of $45^{\circ}$ and the spot area was $0.1 \mathrm{~mm}^{2}$. The deposition time was fixed at 2 hours and the substrate temperature was varied between 25 and $500^{\circ} \mathrm{C}$. The deposition time was 3 min only in the case of films deposited for Transmission Electron Microscopy.

Optical emission spectroscopy (OES) was carried out by a Princeton ICCD device $(1024 \times 1024$ pixels $)$ coupled with a $50 \mathrm{~cm}$ spectrograph (ARC SP-500i). The width of the entrance spectrograph slit was $80 \mu \mathrm{m}$ and the gratings employed were 2400,1200 , and 150 grooves $/ \mathrm{mm}$. The gated system had a time resolution of $2 \mathrm{~ns}$ and each acquisition was integrated over 50 laser shots in order to increase the signalto-noise ratio. Varying the position of the optical elements by a micrometric translation stage, it has been possible to obtain space resolved emission spectra at different distances from the ablated target surface. The same ICCD system, equipped with 105/35 mm quartz Nikkor lens, was used for acquiring gated images of the lateral view of the overall plasma plume emission in the range of $200-800 \mathrm{~nm}$. The acquisition was performed by accumulating up to 20 shots and the spatial resolution achieved by this system was $37 \mu \mathrm{m}$. Both ICCD fast imaging and time resolved spectra were accomplished by delaying the data acquisition of the plasma emission in the range of 10-100,000 ns with respect to the laser pulse. In all cases every data acquisition was carried out on a not previously irradiated surface.

The structure of the films was evaluated by X-ray diffraction (XRD) and by micro-Raman spectroscopy. The XRD measurements were performed by a Philips X'Pert Pro diffractometer, using $\mathrm{Cu} \mathrm{K} \alpha$ radiation. The Raman spectra were recorded in a backscattered configuration using a HORIBA LABRAM HR 800 micro-Raman spectrometer, with $632.8 \mathrm{~nm}$ radiation from $\mathrm{He}-\mathrm{Ne}$ laser source. The deposits' morphology was studied by atomic force microscopy (AFM-Park XE 120) and by a scanning electron microscopy (SEM) apparatus (Philips-FEI ESEM XL30). High resolution Transmission Electron Microscopy (HR-TEM, FeiTECNAI G2 20 TWIN), operating at $200 \mathrm{kV}$ was used to characterize size and distributions of the NPs forming the deposited films. X-ray photoelectron spectra (XPS), acquired by LH-Leybold 100 spectrometer using unmonochromatized $\mathrm{Mg} \mathrm{K} \alpha$ radiation, were used to analyze the films composition and stoichiometry. Wide and detailed spectra were collected in Fixed Analyzer Transmission (FAT) mode with pass energy of $50 \mathrm{eV}$ and channel widths of 1.0 and $0.1 \mathrm{eV}$, respectively. The resolution of the detailed spectra is $1 \mathrm{eV}$.

\section{Results and Discussion}

3.1. Plasma Characterization. The ICCD fast imaging evidences the presence of a plasma plume starting at the end of laser pulse and lasting few hundreds of nanoseconds. This plume is characterized by a front velocity of $4.5 \pm 0.5 \times$ $10^{6} \mathrm{cms}^{-1}$ and an anisotropic angular distribution parameter $n=1.9 . n$ is the cosine exponent in the expression $I(\theta)=$ $I_{0} \cos ^{n} \theta$, where $I(\theta)$ is the flux intensity along a direction forming an angle $\theta$ with the normal to the target surface and $I_{0}$ is the intensity corresponding to $\theta=0$.

OES of the same plume shows many spectral lines assigned to neutral and singly ionized magnesium and boron (Mg I, Mg II, B I, and B II). From the optical emission spectra the time of flight curves for the different species, at 


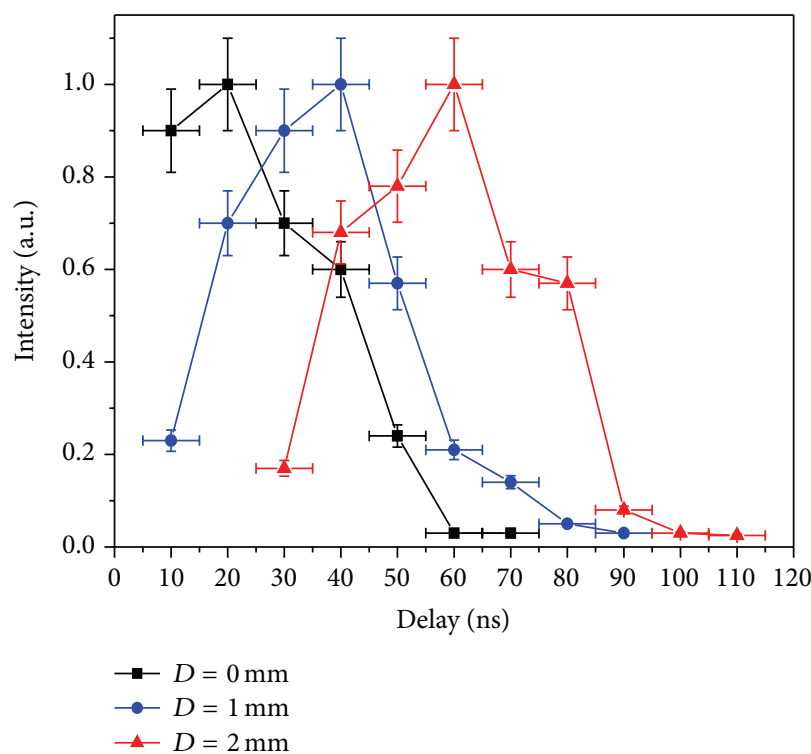

(a)

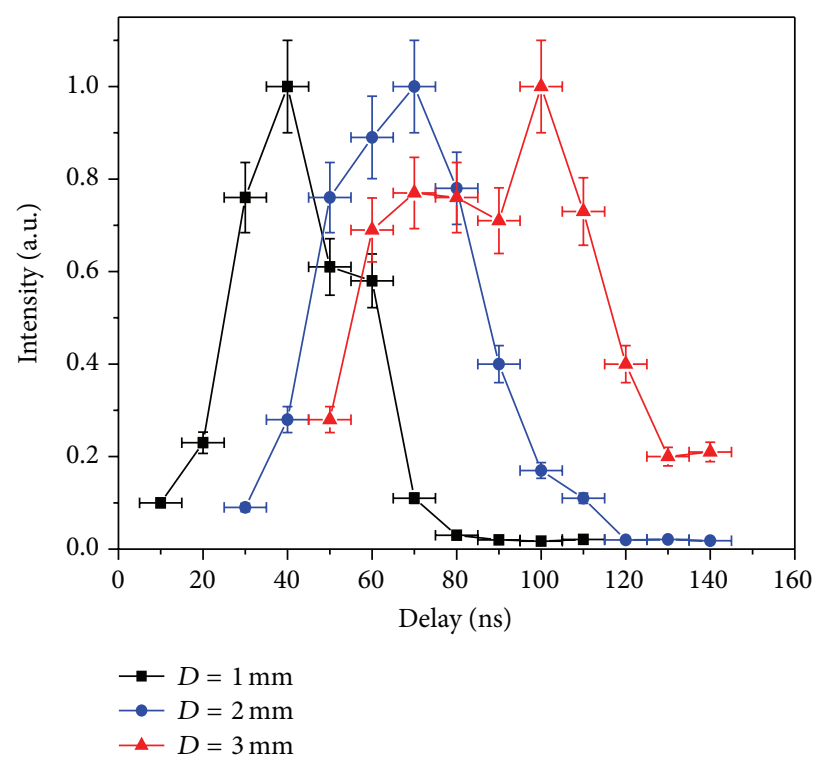

(b)

Figure 1: Normalized time resolved emission plots for (a) B II (412.0 nm) and (b) Mg II (448.0 nm) recorded at different distances from the target. The lines connecting the experimental data were added to facilitate the understanding of the graphics.

different distances from the target, have been obtained and the normalized data for the ions are reported in Figure 1. The data from the time of flight curves give a velocity of $2.5 \pm 0.2 \times 10^{6} \mathrm{cms}^{-1}$ for $\mathrm{Mg} \mathrm{I}, 3.3 \pm 0.3 \times 10^{6} \mathrm{cms}^{-1}$ for Mg II, and $5.0 \pm 0.4 \times 10^{6} \mathrm{cms}^{-1}$ for B II in good agreement with the plume front velocity calculated by ICCD fast imaging. Due to the blazing of our gratings, the intensity of B I peaks was very low and so it was impossible to obtain time of flight curves for this species. Comparing these results with those of the plasmas obtained by ns PLD in Ar atmosphere $[10,33]$, it is evident that the atomic species present in both plasmas are the same except for the absence, in the case of fs ablation, of Mg III, found only by D'Amico et al. in the plasma produced by ns PLD performed by a doubled Nd:YAG laser [10]. This absence is probably related to the lack of interaction between the expanding plume and the laser radiation and to the fact that our experiments have been carried out in vacuum instead of a buffer gas. A comparison of the velocities of the species in ns and fs regimes shows results of the same order of magnitude when we consider a low buffer gas pressure $\left(10^{-2}\right.$ and $0.5 \mathrm{~Pa}$ ) for the ns experiments.

OES data were used for estimating the Mg I excitation temperature $\left(T_{e}\right)$, assuming that the local thermodynamic equilibrium (LTE) condition was fulfilled. Different spectral series, obtained by a fixed detection gate of $25 \mathrm{~ns}$, and starting at delays of $75 \mathrm{~ns}$ from the laser pulse, respectively, were accomplished. $T_{e}$ was determined by using the Boltzmann plot method using the following equation:

$$
\ln \left(\frac{\lambda I}{g A}\right)=\text { const }-\frac{E_{2}}{k_{\mathrm{B}} T_{e}}
$$

where $\lambda$ is the wavelength of the transition line, $I$ is its intensity, $A$ is the transition probability, $E_{2}$ is the energy of the upper state [34], and $k_{\mathrm{B}}$ is the Boltzmann constant.

The results reported in Figure 2(a) show that in the ultrashort laser induced plasma the excitation temperature reaches the value of about $6500 \mathrm{~K}$ during the first nanoseconds and then drops down reaching a plateau temperature of about $4500 \mathrm{~K}$ in about $150 \mathrm{~ns}$.

The temporal behavior of the electron densities induced by the ultrashort laser ablation of the $\mathrm{MgB}_{2}$ target was evaluated by the Full Width at Half Maximum (FWHM) Stark broadening of the $\mathrm{Mg}$ I line centered at $383.8 \mathrm{~nm}$ according to the relation

$$
\begin{aligned}
\Delta \lambda_{1 / 2} & \\
= & 2 W\left(\frac{N_{e}}{10^{16}}\right) \\
& +3.5 A\left(\frac{N_{e}}{10^{16}}\right)^{1 / 4}\left(1-\frac{3}{4} \Lambda^{-1 / 3}\right) W\left(\frac{N_{e}}{10^{16}}\right) A^{\circ},
\end{aligned}
$$

where $W$ and $A^{\circ}$ are the Stark and ion broadening parameters, respectively [35], and $\Lambda$ is the number of particles estimated in the Debye sphere. For nonhydrogenic ions the broadening due to electron contribution is the main one, whereas the perturbation caused by ions is negligible compared to electrons [36]. Intensity and FWHM were estimated by fitting experimental curves with symmetrical Voigt profiles. Different spectral series, obtained by a fixed detection gate of $25 \mathrm{~ns}$, and starting at a delay of $5 \mathrm{~ns}$ from the laser pulse, were accomplished. The results reported in Figure 2(b) show that in the ultrashort induced plasma the electron density starts at a value of about $3.5 \times 10^{17} \mathrm{~cm}^{-3}$ in the first nanoseconds and 


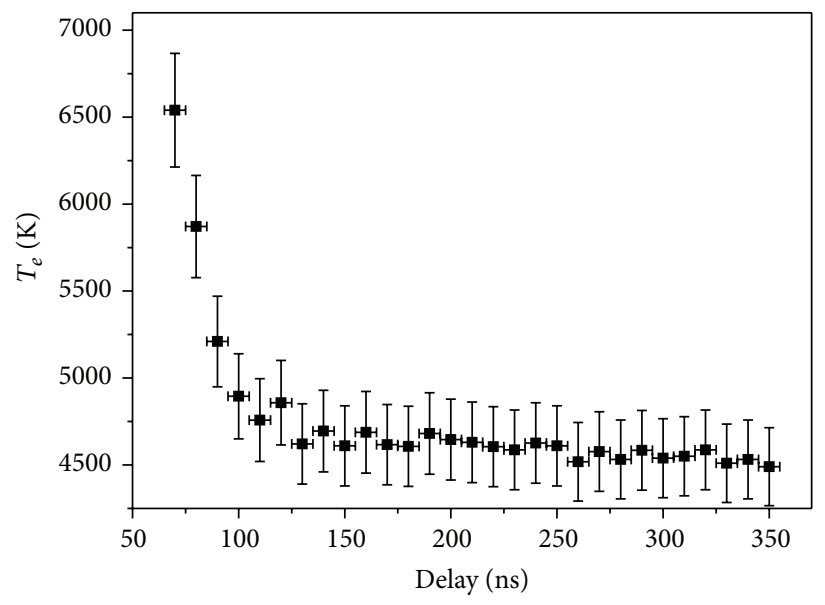

(a)

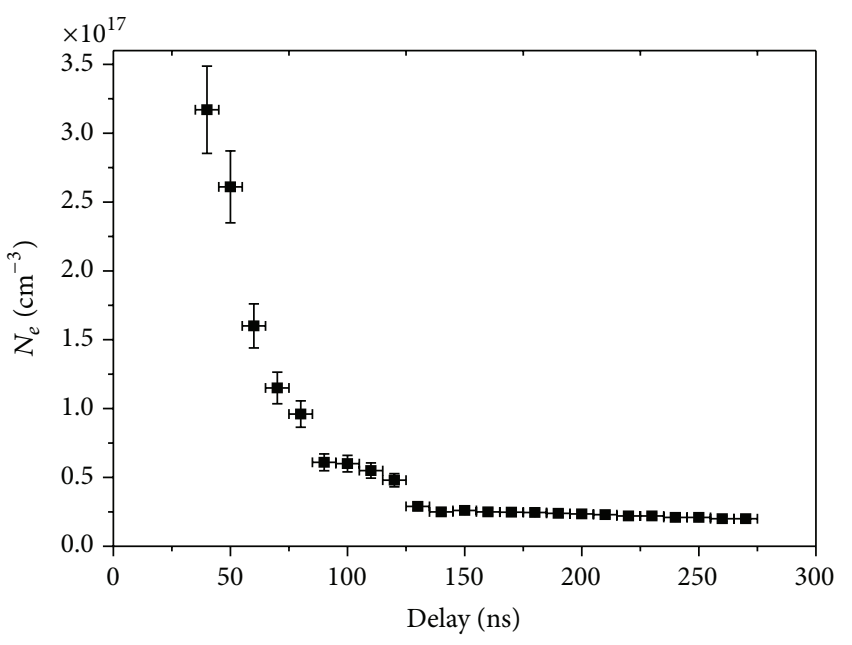

(b)

Figure 2: Temporal evolution of excitation temperature (a) and electron density (b) for magnesium diboride primary plume plasma at a distance of $200 \mu \mathrm{m}$ from the target surface.

then suffers an exponential decay reaching a plateau value of about $1 \times 10^{16} \mathrm{~cm}^{-3}$ after few hundreds of nanoseconds.

After the quenching of the primary plume, one microsecond after the laser pulse, a second emission of material has been observed by ICCD fast imaging. This secondary plume, with a cosine exponent $n=1.1$, has a front velocity of $9.2 \times 10^{4} \mathrm{cms}^{-1}$ and it lasts about $10 \mu \mathrm{s}$. OES achieved in this temporal range shows blackbody-like spectra, suggesting the presence of hot particulate. In order to determine the temperature of the front of the secondary plume at different time delays, its emission in the whole UV-Vis detected range has been fitted by using Wien's equation in the form $I_{\lambda} \propto \varepsilon\left(1 / \lambda^{5}\right) e^{-h c / \lambda k_{\mathrm{B}} T}$ with, in the Mie approximation, the emissivity $\varepsilon$ considered to be proportional to $1 / \lambda$ [37]. The results show an initial temperature of $3490 \pm 10 \mathrm{~K}$ which decreases to $3200 \pm 20 \mathrm{~K}$ after $5 \mu$ s. For longer times the low intensity of the overall emission did not allow temperature calculation.

3.2. Thin Films Characterization. Even if the commercial target is nominally $\mathrm{MgB}_{2}$, XPS analysis shows that the real $\mathrm{B} / \mathrm{Mg}$ ratio is 1.2, evidencing an excess of magnesium, probably due to the synthesis process. On the other hand, XRD analysis of the same target shows the presence of both crystalline $\mathrm{MgB}_{2}$ and $\mathrm{MgB}_{4}$, with the first as the main component and the second presenting only in a small amount. These results are not completely unexpected, since it is known that commercial powders are often nonstoichiometric [38], but, of course, they add further uncertainty to the interpretation of the results obtained in the thin films deposition.

From XRD analyses the films appear to be amorphous in structure, since the spectra show only the presence of the substrate peaks, independently from the substrate temperature. These data are confirmed by micro-Raman spectra that do not evidence the presence of peaks assignable to magnesium borides.
SEM images registered at different deposition temperatures show that the films' morphology does not change with the substrate temperature. In all cases the deposits are uniform and formed by the coalescence of a large number of NPs (Figure 3), as already observed for other metal boride films deposited by ultrashort PLD technique [31]. These NPs are visible in a clearer way in the AFM images reported in Figure 4.

The formation of the films by coalescence of NPs is also confirmed by HR-TEM micrographs, showing the first steps of the film growth (Figure 5). From these images it has been possible to calculate the distribution of the NPs diameters. It is clearly log-normal distribution, the mean value of which is $14 \mathrm{~nm}$ (Figure 6).

XPS analyses performed on films deposited at different temperatures $\left(25^{\circ} \mathrm{C}, 300^{\circ} \mathrm{C}\right.$, and $\left.500^{\circ} \mathrm{C}\right)$ reveal that the films composition varies with the substrate temperature. Since, under the resolution of our XPS apparatus, it is not possible to separate the XPS contribution of magnesium boride from oxide in $\mathrm{Mg} 2 \mathrm{p}$ region (both at about $51 \mathrm{eV}$ ), only the peak-fitting of the $\mathrm{B} 1 \mathrm{~s}$ region is reported. The analysis of the $\mathrm{B}$ 1s region (Figure 7) evidences, in the case of films deposited at 300 and $500^{\circ} \mathrm{C}$, that three contributions at 187.4, 189.7, and $192.0 \mathrm{eV}$ are present. These contributions are assigned, following the literature indications $[39,40]$, to magnesium-bonded boron, atomic boron, and boron oxide $\left(\mathrm{B}_{2} \mathrm{O}_{3}\right)$, respectively. In the case of the target and of films deposited at room temperature, only two peaks are present, assigned to magnesium bonded boron $(187.0 \mathrm{eV})$ and to boron oxide $(192.3 \mathrm{eV})$. Considering the difficulty of separating the contributions of magnesium boride from oxide, the correct stoichiometry of the magnesium boride films is not so easy to establish. By comparing the molar ratios obtained from XPS data for the films with those obtained for the target (Table 1), some considerations can be done. First of all, a large loss of $\mathrm{Mg}$ in the films, in particular in the films deposited at 300 and 


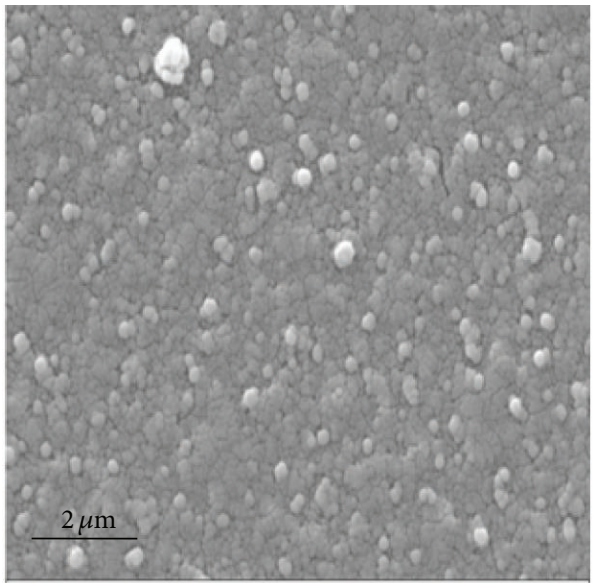

(a)

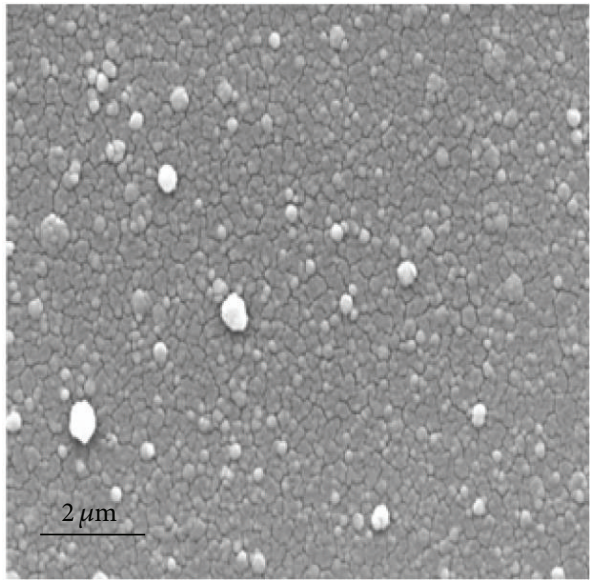

(b)

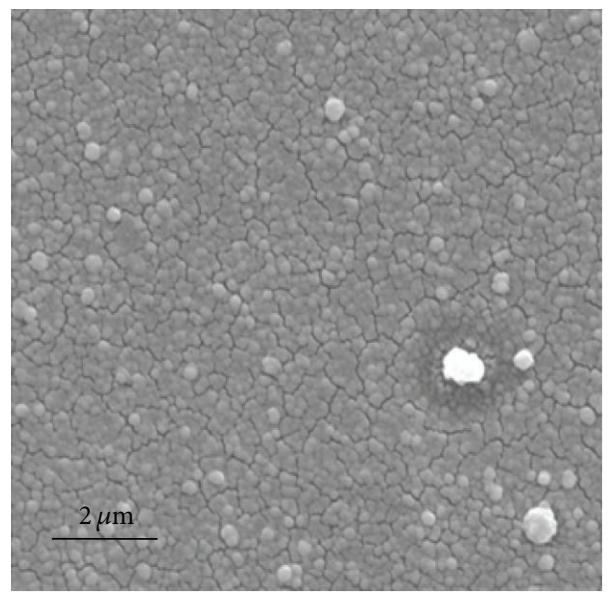

(c)

Figure 3: SEM micrographs of films deposited at different substrate temperatures: (a) $25^{\circ} \mathrm{C}$, (b) $300^{\circ} \mathrm{C}$, and (c) $500^{\circ} \mathrm{C}$. The deposition time was 2 hours for all samples.

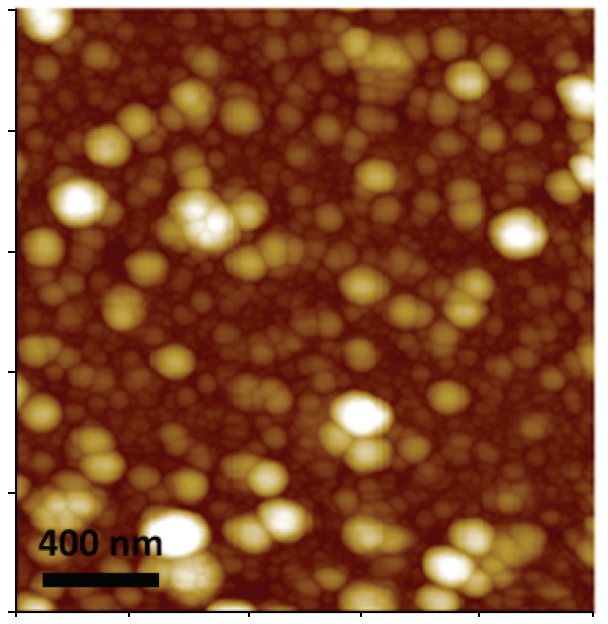

(a)

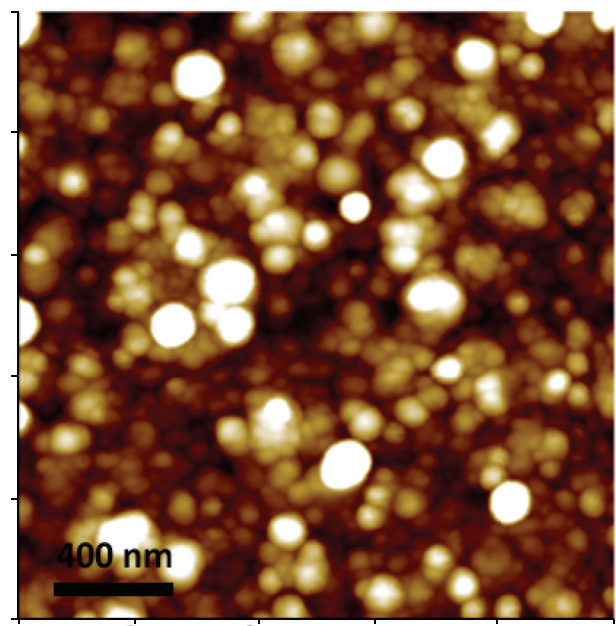

(b)

FIGURE 4: AFM images of films deposited at a substrate temperature of $25^{\circ} \mathrm{C}$ (a) and $500^{\circ} \mathrm{C}$ (b). In both cases the deposition time was 2 hours. 


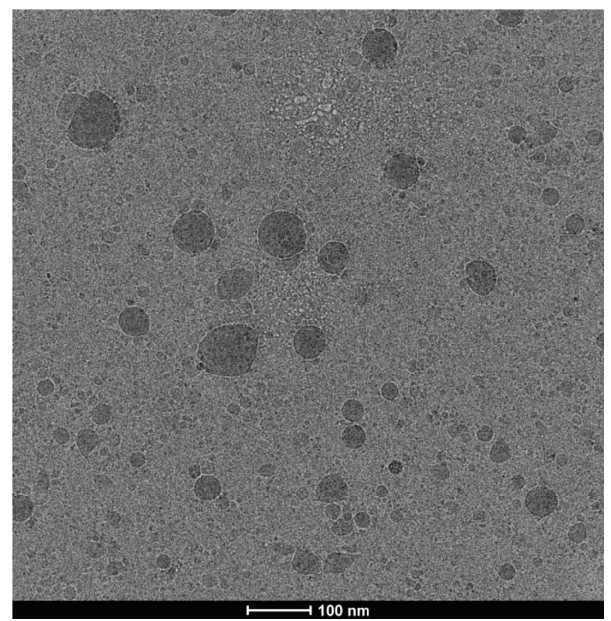

FIGURE 5: TEM micrograph showing the initial steps of the films formation.

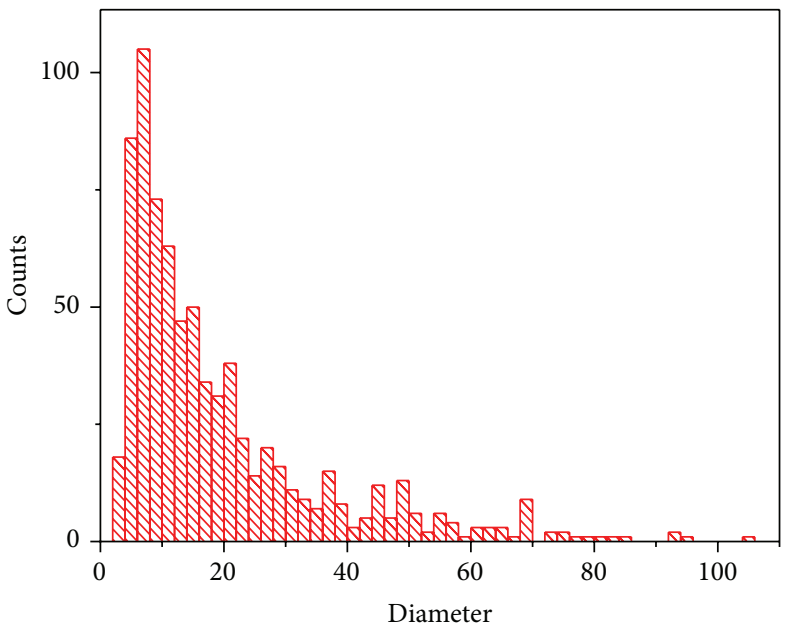

(a)

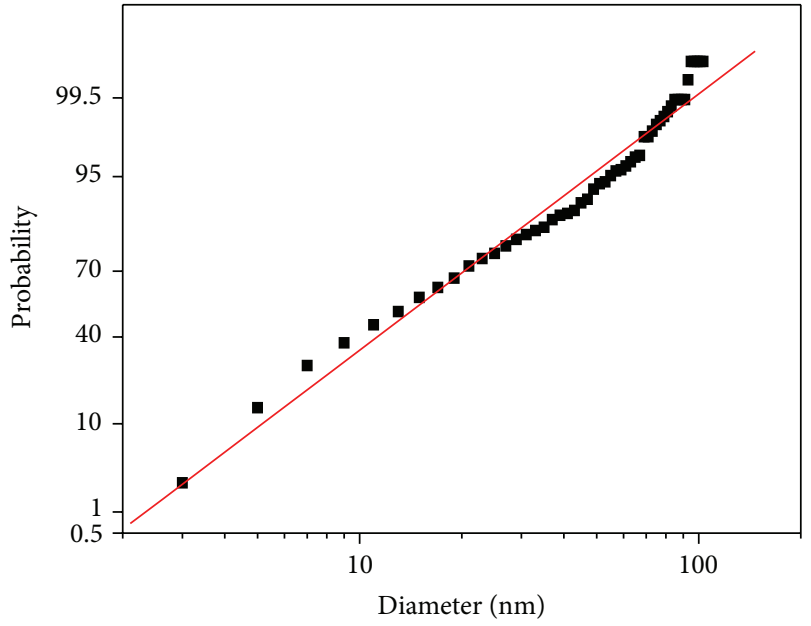

(b)

FIGURE 6: (a) Size distribution of NPs deposited by fs PLD of the magnesium diboride target. The size distribution has been obtained by analysis of more than 500 particles from TEM images; (b) diameter-probability plot for the NPs size distribution from TEM images. The solid line fit represents the log-normal size distribution. The value at $50 \%$ probability gives the mean diameter of the particles.

$500^{\circ} \mathrm{C}$, is evident. If we consider the total $\mathrm{B}_{\text {boride }} / \mathrm{Mg}_{\text {total }}$ ratio in the films we see that the larger quantity of $\mathrm{Mg}$ is present in the film deposited at room temperature $\left(\mathrm{B}_{\text {boride }} / \mathrm{Mg}_{\text {total }}=\right.$ 1.7). Of course, not all the magnesium present in the film will be bonded to boron (e.g., in the $\mathrm{MgB}_{2}$ target, the ratio between boron-bonded and non-boron-bonded magnesium is approximately $1 / 1.5$, supposing that the boride forming the target is stoichiometric) so the stoichiometry of the film deposited at $25^{\circ} \mathrm{C}$ will probably be $\mathrm{MgB}_{y}$ with $y$ comprised between 2 and 4 . This should mean a mixture of $\mathrm{MgB}_{2}$ and $\mathrm{MgB}_{4}$. For higher substrate temperatures, further depletion of magnesium is evident in the films, with the occurrence of atomic boron. The large presence of boron oxide in both target and films could be due to surface oxidation taking place in the presence of atmospheric oxygen and is clearly a surface phenomenon, since no boron oxide peaks were found in the XRD spectrum of the target.
TABLE 1: Molar ratios obtained from XPS data for the target and the deposited films.

\begin{tabular}{|c|c|c|c|c|}
\hline & Target & Film, $25^{\circ} \mathrm{C}$ & Film, $300^{\circ} \mathrm{C}$ & Film, $500^{\circ} \mathrm{C}$ \\
\hline $\mathrm{B}_{\text {boride }} / \mathrm{Mg}_{\text {total }}$ & $0.8 \pm 0.1$ & $1.7 \pm 0.2$ & $2.9 \pm 0.3$ & $3.0 \pm 0.3$ \\
\hline $\mathrm{B}_{\text {total }} / \mathrm{Mg}_{\text {total }}$ & $1.2 \pm 0.1$ & $2.7 \pm 0.3$ & $4.5 \pm 0.5$ & $4.3 \pm 0.4$ \\
\hline
\end{tabular}

3.3. Ablation-Deposition Mechanism. From the data obtained by the analyses of the two plumes produced by fs ablation of $\mathrm{MgB}_{2}$, it is evident that the characteristics of the primary plume are similar to those of the plasmas produced by the ablation of the same target performed by ns laser sources $[10,33]$. Indeed, as already seen, the atomic species are the same, while their velocities are comparable. Due to the lack of literature data about the excitation temperature and the electron density of the plasmas produced by ns laser ablation, 


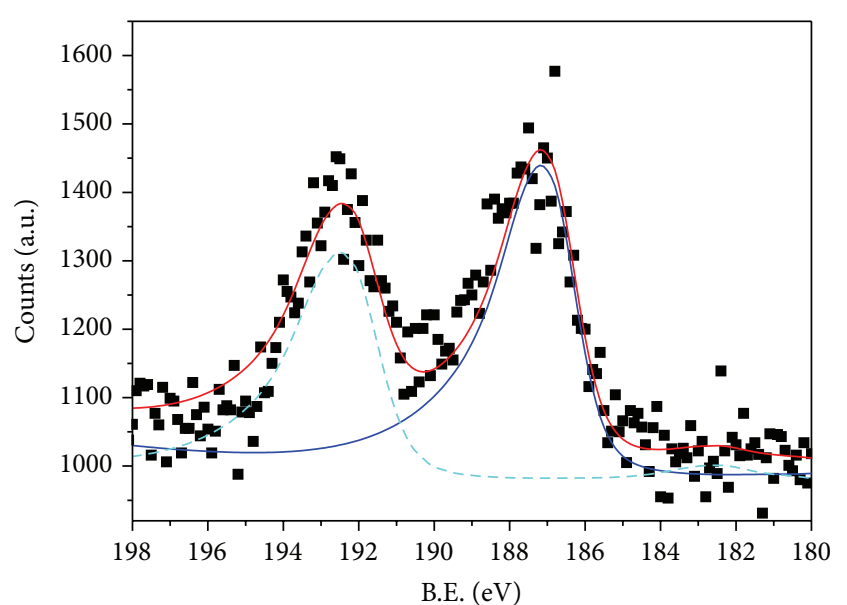

(a)

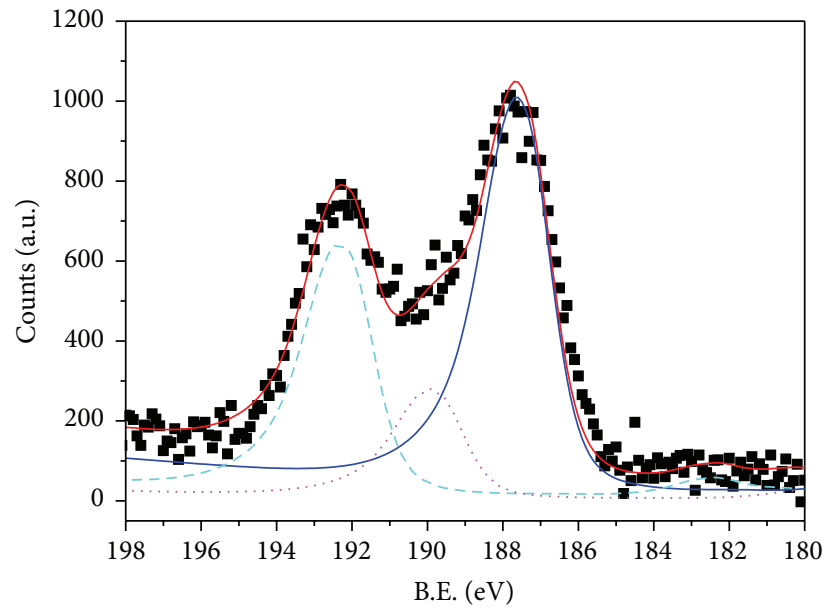

(c)

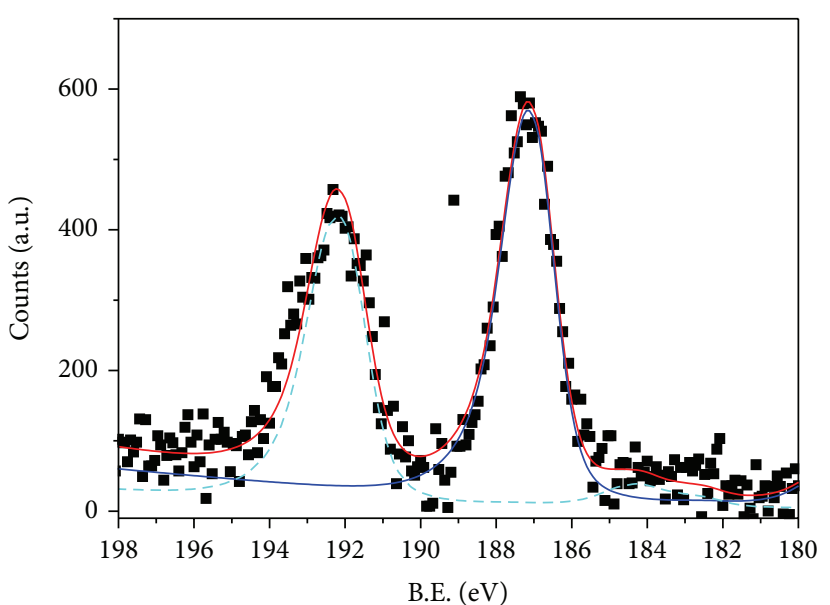

(b)

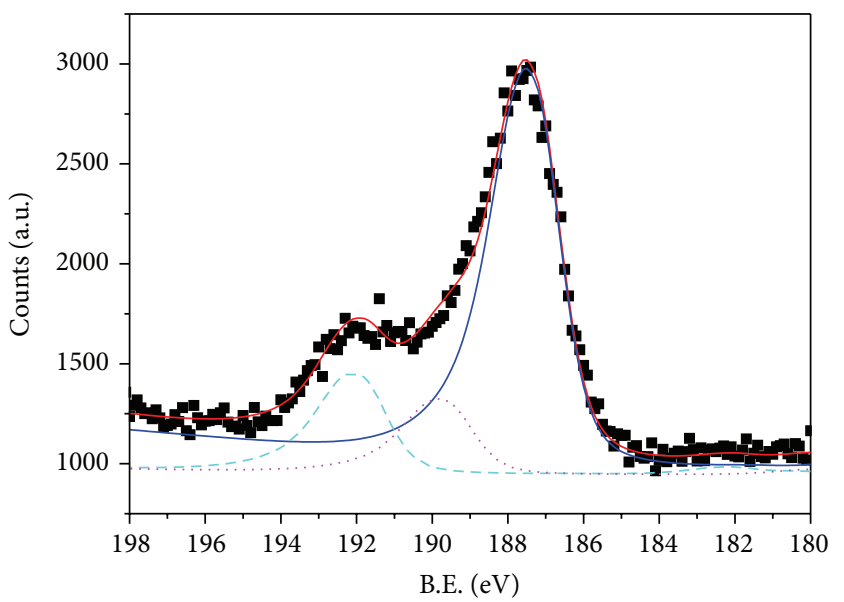

(d)

Figure 7: XPS spectra of the $\mathrm{B} 1 \mathrm{~s}$ region of (a) $\mathrm{MgB}_{2}$ target, (b) film deposited at $25^{\circ} \mathrm{C}$, (c) film deposited at $300^{\circ} \mathrm{C}$, and (d) film deposited at $500^{\circ} \mathrm{C} . \mathrm{MgB}_{y}$ (blue solid line); Boron (magenta dotted line); $\mathrm{B}_{2} \mathrm{O}_{3}$ (cyan dashed line).

it is impossible to verify whether these parameters match those reported in this paper for fs ablation but, considering the results from a paper where such comparisons were made, even if on other systems [41], we can hypothesize also in this case a similarity. On the other hand, the characteristics of the deposited films are very different in the two regimes. Since the fs primary plume is very similar to the single plume formed by ns ablation, the different films structure and composition should be related to the presence, in fs ablation, of a secondary plume. As already seen, this plume is formed by NPs. These NPs, probably ejected directly from the target, represent the main component of the ablated material and are responsible for the formation of a nanostructured film $[8,24]$. In the case of $\mathrm{MgB}_{2}$ PLD, the reported experimental results can be interpreted considering an ablation-deposition mechanism already proposed for other systems deposited by ultrashort PLD [28, 31, 32]. In those previous works we have proposed a mechanism considering the fact that the NPs, forming the secondary plume, were ejected directly from the target and presented initially the target stoichiometry and all the composition changes took place during their flight to the substrate. Indeed, the composition changes depend on the different equilibrium vapor pressures of the elements forming the NPs. From this point of view magnesium diboride, with the large difference of vapor pressure between magnesium and boron, is a good system to test the hypothesis. As previously reported, the initial temperature of the particles was about $3500 \mathrm{~K}$ and it decreased to 3200 after $2 \mu \mathrm{s}$. In these conditions, considering the Mg-B phase diagram [42], the system is formed by a liquid phase containing mainly boron in equilibrium with a gaseous phase containing mainly magnesium. During the flight to the substrate, which lasts about $20 \mu \mathrm{s}$, the NPs will experience loss of magnesium, leading to a final composition consisting, according again to the $\mathrm{Mg}-\mathrm{B}$ phase diagram, of a mixture of $\mathrm{MgB}_{2}$ and $\mathrm{MgB}_{4}$. It should be pointed out that probably the presence in the target of a large excess of magnesium could partly compensate the loss of the same element during the flight of the NPs.

Of course, due to the high vapor pressure of $\mathrm{Mg}$, the heating of the substrate will lead to a further decrease of 
the concentration of this element in the deposited films. In fact, the films with the higher $\mathrm{Mg}$ concentration were those deposited at room temperature. On the other hand, the increase of the substrate temperature did not improve the films' crystallinity and eventually resulted to be counterproductive.

\section{Conclusions}

In conclusion, the laser ablation of the magnesium diboride target performed by an ultrashort pulse laser has confirmed the importance of the nanoparticles forming the secondary plume in the deposition of thin films and the low importance of the species forming the primary plume. Indeed, the films are formed by the coalescence of a large number of NPs and their composition can be related to the dynamics of melted NPs ejected directly from the target. The films composition depends on the NPs composition and on the substrate temperature. From this point of view, the best films have been obtained at room temperature, since heating of the substrate increases the loss of magnesium and does not improve the film's crystallinity.

\section{Conflict of Interests}

The authors declare that there is no conflict of interests regarding the publication of this paper.

\section{References}

[1] S. Carenco, D. Portehault, C. Boissière, N. Mézailles, and C. Sanchez, "Nanoscaled metal borides and phosphides: recent developments and perspectives," Chemical Reviews, vol. 113, no. 10, pp. 7981-8065, 2013.

[2] J. Nagamatsu, N. Nakagawa, T. Muranaka, Y. Zenitani, and J. Akimitsu, "Superconductivity at $39 \mathrm{~K}$ in magnesium diboride," Nature, vol. 410, no. 6824, pp. 63-64, 2001.

[3] X. X. Xi, " $\mathrm{MgB}_{2}$ thin films," Superconductor Science and Technology, vol. 22, no. 4, Article ID 043001, 15 pages, 2009.

[4] E. W. Collings, M. D. Sumption, and T. Tajima, "Magnesium diboride superconducting RF resonant cavities for high energy particle acceleration," Superconductor Science and Technology, vol. 17, no. 9, pp. S595-S601, 2004.

[5] L. D. Cooley, C. B. Eom, E. E. Hellstrom, and D. C. Larbalestier, "Potential application of magnesium diboride for accelerator magnet applications," in Proceedings of the Particle Accelerator Conference (PAC '01), vol. 1, pp. 203-207, IEEE, Chicago, Ill, USA, 2001.

[6] Y. Iwasa, D. C. Larbalestier, M. Okada, R. Penco, M. D. Sumption, and X. X. Xi, "A round table discussion on MgB2 toward a wide market or a niche production? A summary," IEEE Transactions on Applied Superconductivity, vol. 16, no. 2, pp. 14571464, 2006.

[7] R. Eason, Ed., Pulsed Laser Deposition of Thin Films: Applications-Led Growth of Functional Materials, Wiley-Interscience, 2007.

[8] P. Balling and J. Schou, "Femtosecond-laser ablation dynamics of dielectrics: basics and applications for thin films," Reports on Progress in Physics, vol. 76, no. 3, Article ID 036502, 39 pages, 2013.
[9] G. Grassano, W. Ramadan, Ferrando V et al., "As-grown magnesium diboride superconducting thin films deposited by pulsed laser deposition," Superconductor Science and Technology, vol. 14, no. 9, pp. 762-764, 2001.

[10] G. D’Amico, A. Di Trolio, A. Morone, S. Orlando, and A. Santagata, "Optical characterization of magnesium diboride plasma plume induced by pulsed laser ablation," Applied Surface Science, vol. 208-209, no. 1, pp. 96-100, 2003.

[11] J. Kim, R. K. Singh, N. Newman, and J. M. Rowell, "Thermochemistry of $\mathrm{MgB}_{2}$ thin film synthesis," IEEE Transactions on Applied Superconductivity, vol. 13, no. 2, pp. 3238-3241, 2003.

[12] W. N. Kang, H.-J. Kim, E.-H. Choi, C. U. Jung, and S.-I. Lee, " $\mathrm{MgB}_{2}$ superconducting thin films with a transition temperature of 39 kelvin," Science, vol. 292, no. 5521, pp. 1521-1523, 2001.

[13] S. R. Shinde, S. B. Ogale, R. L. Greene et al., "Superconducting MgB2 thin films by pulsed laser deposition," Applied Physics Letters, vol. 79, no. 2, pp. 227-229, 2001.

[14] D. H. A. Blank, H. Hilgenkamp, A. Brinkman, D. Mijatovic, G. Rijnders, and H. Rogalla, "Superconducting Mg-B films by pulsed-laser deposition in an in situ two-step process using multicomponent targets," Applied Physics Letters, vol. 79, no. 3, pp. 394-396, 2001.

[15] A. Berenov, Z. Lockman, X. Qi et al., "Growth of strongly biaxially aligned $\mathrm{MgB}_{2}$ thin films on sapphire by postannealing of amorphous precursors," Applied Physics Letters, vol. 79, no. 24, pp. 4001-4003, 2001.

[16] X. H. Zeng, A. Sukiasyan, X. X. Xi et al., "Superconducting properties of nanocrystalline $\mathrm{MgB}_{2}$ thin films made by an in situ annealing process," Applied Physics Letters, vol. 79, no. 12, pp. 1840-1842, 2001.

[17] H. M. Christen, H. Y. Zhai, C. Cantoni et al., "Superconducting magnesium diboride films with $T_{c} \approx 24 \mathrm{~K}$ grown by pulsed laser deposition with in situ anneal," Physica C: Superconductivity, vol. 353, no. 3-4, pp. 157-161, 2001.

[18] H. Y. Zhai, H. M. Christen, L. Zhang et al., "Superconducting magnesium diboride films on $\mathrm{Si}$ with $T_{c 0} \sim 24$ grown via vacuum annealing from stoichiometric precursors," Applied Physics Letters, vol. 79, no. 16, pp. 2603-2605, 2001.

[19] C. Ferdeghini, V. Ferrando, G. Grassano et al., "Transport properties of c-oriented $\mathrm{MgB}_{2}$ thin films grown by pulsed laser deposition," Physica C: Superconductivity, vol. $372-376$, no. 2, pp. 1270-1273, 2002.

[20] Y. Hikita, T. Fukumura, T. Ito, M. Kawasaki, and H. Takagi, "Fabrication of $\mathrm{MgB}_{2}$ thin film on boride substrates by pulsed laser deposition," Journal of Low Temperature Physics, vol. 131, no. 5-6, pp. 1187-1191, 2003.

[21] V. Ferrando, S. Amoruso, E. Bellingeri et al., "Growth methods of c-axis oriented $\mathrm{MgB}_{2}$ thin films by pulsed laser deposition," Superconductor Science and Technology, vol. 16, no. 2, pp. 241245, 2003.

[22] T. Uchiyama, H. Koga, and I. Iguchi, "In situ growth of superconducting $\mathrm{MgB}_{2}$ thin films using a melting process," Japanese Journal of Applied Physics, vol. 43, no. 1, pp. 121-123, 2004.

[23] G. O’Connell, T. Donnelly, and J. G. Lunney, "Nanoparticle plume dynamics in femtosecond laser ablation of gold," Applied Physics A: Materials Science and Processing, vol. 117, no. 1, pp. 289-293, 2014.

[24] N. Tsakiris, K. K. Anoop, G. Ausanio et al., "Ultrashort laser ablation of bulk copper targets: dynamics and size distribution of the generated nanoparticles," Journal of Applied Physics, vol. 115, no. 24, Article ID 243301, 10 pages, 2014. 
[25] D. Perez and L. J. Lewis, "Molecular-dynamics study of ablation of solids under femtosecond laser pulses," Physical Review B, vol. 67, no. 18, Article ID 184102, 2003.

[26] D. Grojo, J. Hermann, and A. Perrone, "Plasma analyses during femtosecond laser ablation of Ti, Zr, and Hf," Journal of Applied Physics, vol. 97, no. 6, Article ID 063306, 9 pages, 2005.

[27] L. J. Lewis and D. Perez, "Laser ablation with short and ultrashort laser pulses: basic mechanisms from moleculardynamics simulations," Applied Surface Science, vol. 255, no. 10, pp. 5101-5106, 2009.

[28] R. Teghil, L. D’Alessio, A. De Bonis et al., "Ultra-short pulse laser ablation of $\mathrm{Al}_{70} \mathrm{Cu}_{20} \mathrm{Fe}_{10}$ alloy: nanoparticles generation and thin films deposition," Thin Solid Films, vol. 517, no. 6, pp. 18801886, 2009.

[29] S. Besner and M. Meunier, "Laser synthesis of nanomaterials," in Laser Precision Microfabrication, K. Sugioka, M. Meunier, and A. Piqué, Eds., vol. 135, pp. 163-188, Springer, Berlin, Germany, 2010.

[30] J. Perrière, C. Boulmer-Leborgne, R. Benzerga, and S. Tricot, "Nanoparticle formation by femtosecond laser ablation," Journal of Physics D: Applied Physics, vol. 40, no. 22, pp. 7069-7076, 2007.

[31] A. De Bonis, A. Santagata, J. V. Rau et al., "Two-phase zirconium boride thin film obtained by ultra-short pulsed laser ablation of a $\mathrm{ZrB}_{12}$ target," Applied Surface Science, vol. 283, pp. 715-721, 2013.

[32] R. Teghil, L. D’Alessio, A. De Bonis et al., "Nanoparticles and thin film formation in ultrashort pulsed laser deposition of vanadium oxide," Journal of Physical Chemistry A, vol. 113, no. 52, pp. 14969-14974, 2009.

[33] S. Amoruso, M. Armenante, R. Bruzzese et al., "Pressure effects during excimer laser ablation of magnesium diboride targets," Applied Surface Science, vol. 208-209, no. 1, pp. 39-44, 2003.

[34] NIST, NIST Database, National Institute of Standards and Technology, Gaithersburg, Md, USA, 2003, http://www.nist.gov.

[35] S. Djeniže, S. Bukvić, and A. Srećković, "Stark broadening and transition probability ratios in the Mg I spectrum," Astronomy and Astrophysics, vol. 425, no. 1, pp. 361-365, 2004.

[36] S. S. Harilal, C. V. Bindhu, R. C. Issac, V. P. N. Nampoori, and C. P. G. Vallabhan, "Electron density and temperature measurements in a laser produced carbon plasma," Journal of Applied Physics, vol. 82, no. 5, pp. 2140-2146, 1997.

[37] C. F. Bohren and D. R. Huffman, Absorption and Scattering of Light by Small Particles, Wiley, Chichester, UK, 1983.

[38] C. E. J. Dancer, D. Prabhakaran, A. Crossley, R. I. Todd, and C. R. M. Grovenor, "Effects of attrition and ball milling on the properties of magnesium diboride," Superconductor Science and Technology, vol. 23, no. 6, 2010.

[39] L. Crodani, G. Rossetto, S. Kaciulis, A. Mezzi, N. El-Habra, and V. Palmieri, "Study of magnesium boride films obtained from $\mathrm{Mg}\left(\mathrm{BH}_{4}\right)_{2}$ by CVD," Chemical Vapor Deposition, vol. 13, no. 8, pp. 414-419, 2007.

[40] NIST X-Ray Photoelectron Spectroscopy Database, Version 3.5, National Institute of Standards and Technology, Gaithersburg, Md, USA, 2003, http://srdata.nist.gov/xps/.

[41] A. De Bonis, A. Galasso, V. Marotta et al., "Pulsed laser ablation of indium tin oxide in the nano and femtosecond regime: characterization of transient species," Applied Surface Science, vol. 252, no. 13, pp. 4632-4636, 2006.

[42] H. Okamoto, "B-Mg (boron-magnesium)," Journal of Phase Equilibria, vol. 24, article 92, 2003. 

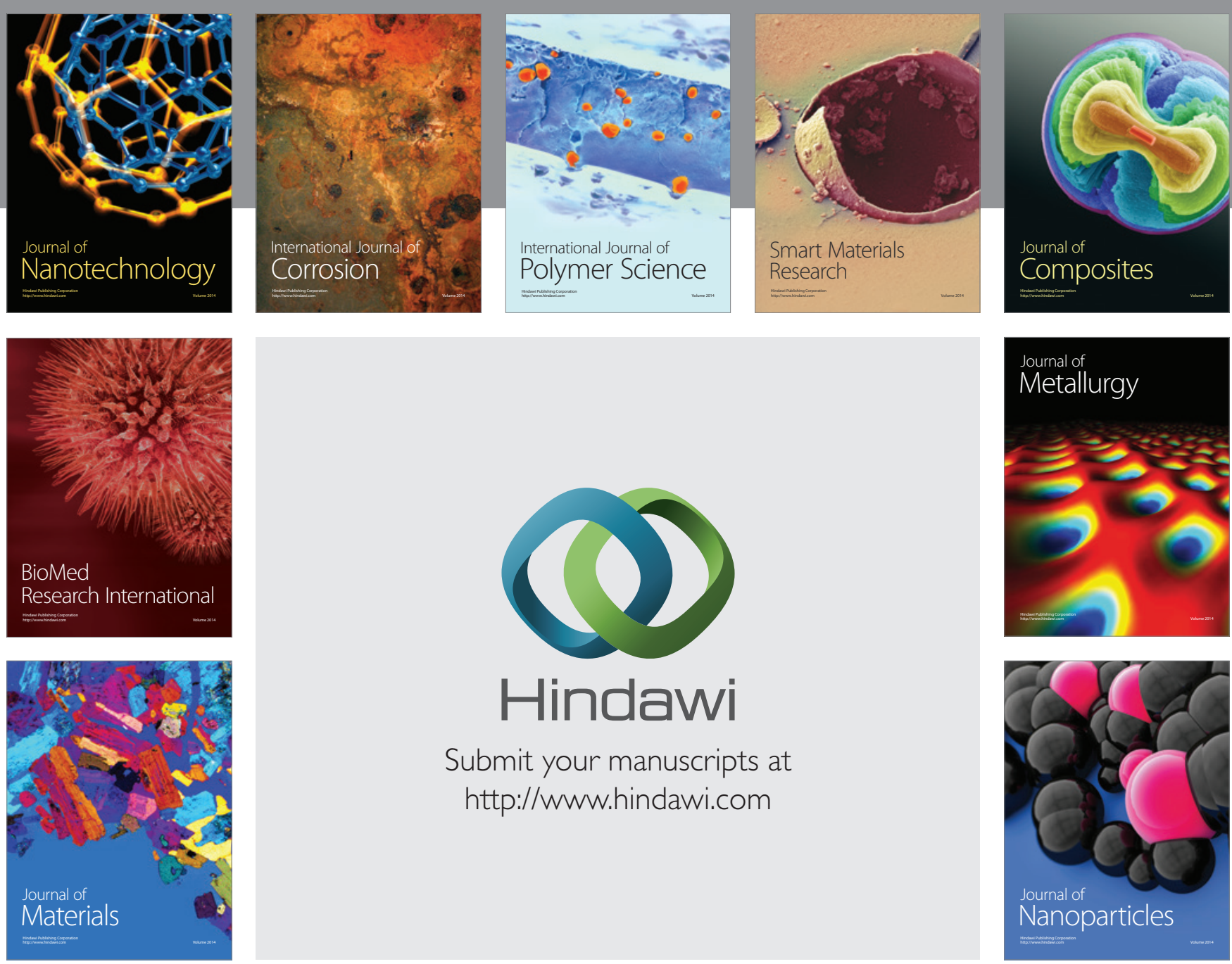

Submit your manuscripts at http://www.hindawi.com
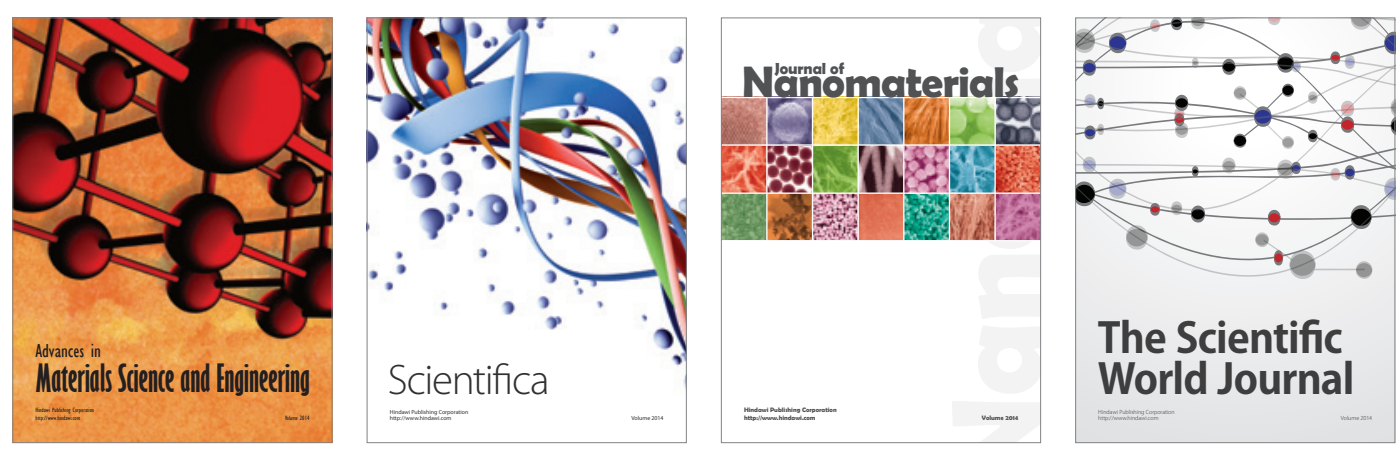

\section{The Scientific World Journal}
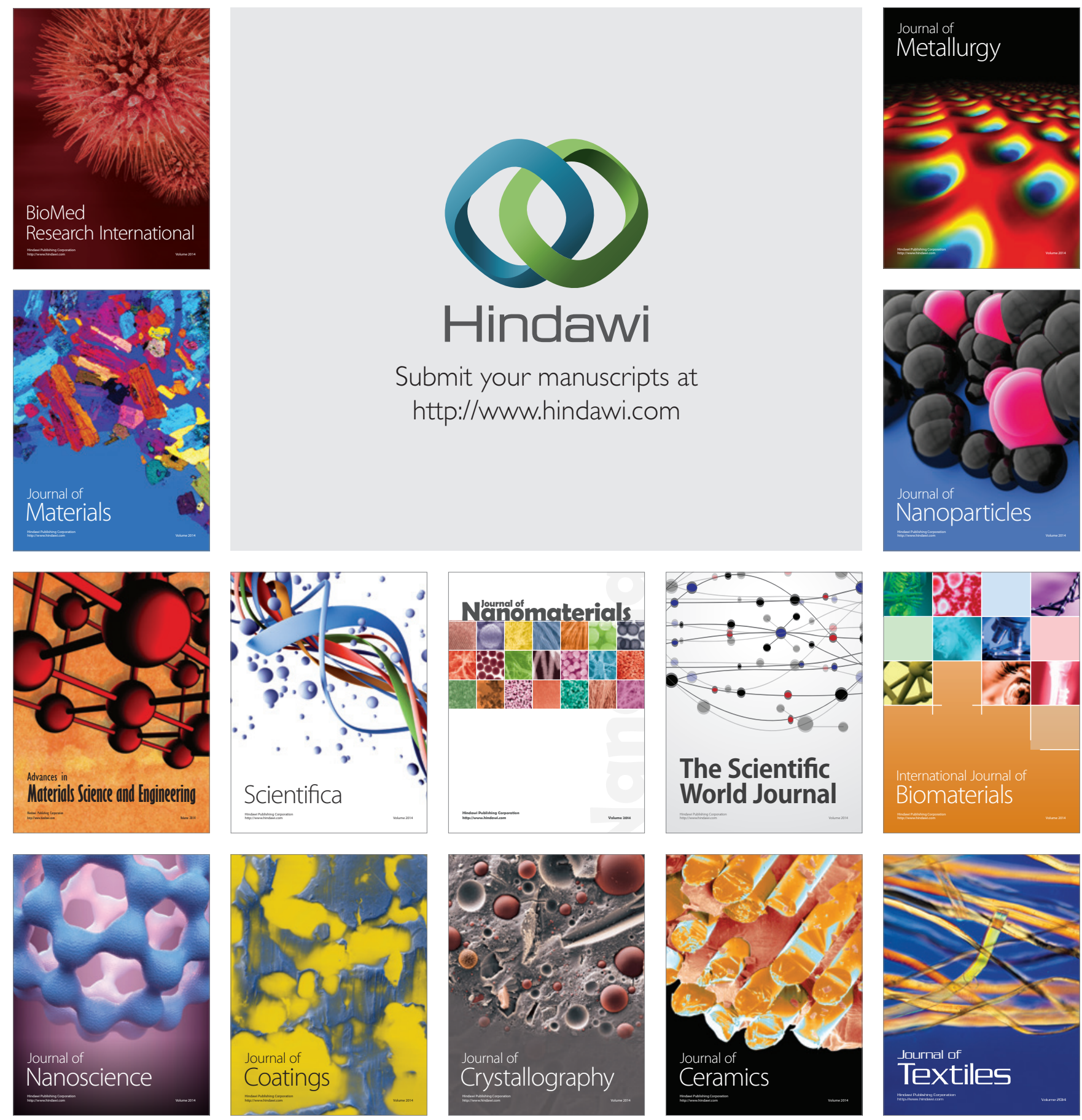